\title{
Towards Magnetic Bimetallic Wire-Like Nanostructures Magnetic Field as Growth Parameter
}

\author{
M. Krajewski ${ }^{a, *}$, M. TokArczyK ${ }^{b}$, A. Witecka $^{a}$, S. LeWińskA $^{c}$, \\ A. ŚlawsKa-WANIEWSKA ${ }^{c}$ AND M. PŁOCIŃSKA ${ }^{d}$ \\ ${ }^{a}$ Institute of Fundamental Technological Research, Polish Academy of Sciences, \\ A. Pawińskiego 5B, 02-106 Warsaw, Poland \\ ${ }^{b}$ University of Warsaw, Faculty of Physics, L. Pasteura 5, 02-093 Warsaw, Poland \\ ${ }^{c}$ Institute of Physics, Polish Academy of Sciences, Aleja Lotnikow 32/46, PL-02668 Warsaw, Poland \\ ${ }^{d}$ Warsaw University of Technology, Faculty of Materials Science and Engineering, \\ Wołoska 141, 02-507 Warsaw, Poland
}

\begin{abstract}
The magnetically-assisted growth of the amorphous bimetallic iron-nickel wire-like nanostructures is presented in this work. The applied process is based on a simple reduction reaction of aqueous solutions containing $\mathrm{Fe}^{2+}$ and $\mathrm{Ni}^{2+}$ ions with $\mathrm{NaBH}_{4}$ in the presence of an external magnetic field of about $0.05 \mathrm{~T}$. The morphology, chemical composition, and magnetic properties of as-prepared $\mathrm{Fe}-\mathrm{Ni}$ nanostructures have been determined by means of scanning electron microscopy, transmission electron microscopy, energy-dispersive X-ray spectroscopy, X-ray diffractometry, and vibrating sample magnetometry. The obtained experimental data indicate that the as-prepared samples exhibit quite complex architectures i.e., they comprise of nanoparticles aligned in almost straight lines. In addition, they reveal the typical core-shell structures where the amorphous bimetallic alloy cores are covered by thin amorphous oxide shells. In turn, the magnetic measurements show that the $\mathrm{Fe}-\mathrm{Ni}$ wire-like nanostructures behave as typical ferromagnetic nanomaterials and their magnetic parameters like saturation magnetizations and coercivities are strictly dependent on their sizes and chemical compositions.
\end{abstract}

DOI: 10.12693/APhysPolA.137.59

PACS/topics: amorphous materials, bimetallic nanostructures, magnetic-field-induced synthesis, magnetic materials, wire-like nanostructures

\section{Introduction}

For ages, the magnetic field has been recognized as either an intrinsic material property or a parameter which describes the magnetic interactions between materials. However, in the end of 20th century it was demonstrated that the magnetic field can also act as a reaction parameter, similar to conventional reaction conditions, i.e., temperature, pressure, time, and chemical additives. This becames a milestone in the field of advanced engineering of the magnetic wire-like nanostructures [1].

The magnetic-field-induced (MFI) synthesis is a relatively new inexpensive approach which allows producing various metallic wire-like nanomaterials like cobalt nanowires [2], nickel nanowires [3], and iron nanowires [4]. However, it is hard to apply this technique in order to manufacture the bimetallic wire-like nanostructures. This is mainly associated with the fact that the synthesis of such nanomaterials is much more complicated than the simple metallic structures. Facing this challenging task, we proposed in this work the MFI process, which leads to the formation of bimetallic ironnickel $(\mathrm{Fe}-\mathrm{Ni})$ nanochains with desired iron-to-nickel

\footnotetext{
*corresponding author; e-mail: mkraj@ippt.pan.pl
}

ratios. Besides that, their morphological, structural, and magnetic characterizations are also discussed.

\section{Experimental}

The following chemical reagents were used in this work: iron(II) chloride hydrate $\left(\mathrm{FeCl}_{2} \cdot x \mathrm{H}_{2} \mathrm{O}, 98 \%\right.$, Carl Roth $\mathrm{GmbH})$, cobalt(II) chloride hexahydrate $\left(\mathrm{CoCl}_{2} \cdot 6 \mathrm{H}_{2} \mathrm{O}\right.$, $97 \%$, Carl Roth $\mathrm{GmbH})$, sodium borohydride $\left(\mathrm{NaBH}_{4}\right.$, 97\%, Carl Roth $\mathrm{GmbH}$ ), acetone (99.5\%, Carl Roth $\mathrm{GmbH}$ ), and ethanol (99.8\%, Avantor). All of them were of analytic grade and were used as received without further purification.

In the manufacturing process proposed in this work, the bimetallic $\mathrm{Fe}-\mathrm{Ni}$ wire-like nanostructures were synthesised according to Eq. (1):

$$
\begin{gathered}
x \mathrm{FeCl}_{2}+(1-x) \mathrm{NiCl}_{2}+2 \mathrm{NaBH}_{4}+6 \mathrm{H}_{2} \mathrm{O} \rightarrow \\
\mathrm{Fe}_{x} \mathrm{Ni}_{1-x} \downarrow+2 \mathrm{H}_{3} \mathrm{BO}_{3}+2 \mathrm{NaCl}+7 \mathrm{H}_{2} \uparrow,
\end{gathered}
$$

where $x$ equalled 0.75 or 0.25 . This enabled us producing the nanomaterials with the $\mathrm{Fe}_{0.75} \mathrm{Ni}_{0.25}$ and $\mathrm{Fe}_{0.25} \mathrm{Ni}_{0.75}$ chemical compositions. Moreover, the process was carried out in an extremal magnetic field of $0.05 \mathrm{~T}$. In fact, this concept combines two well-known approaches, namely (i) the chemical reduction with $\mathrm{NaBH}_{4}$ as a reducing agent, which was used before to produce the air-stable $\mathrm{Fe}-\mathrm{Ni}$ nanoparticles [5], and (ii) the application of the extremal magnetic field, which served as a growth parameter [1]. 
After the synthesis, the obtained products were washed three times with ethanol and three times with acetone in order to expel the reaction by-products. Finally, they were dried in a vacuum dryer at $50^{\circ} \mathrm{C}$ for $2 \mathrm{~h}$.

The as-prepared Fe-Ni nanomaterials were characterized by means of a Hitachi SU8000 scanning electron microscope (SEM), a JEOL JEM 2100 FEG high resolution transmission electron microscope (HRTEM) equipped with an Oxford energy dispersive X-ray spectrometer (EDS), a Phillips X'Pert Diffractometer (XRD) equipped with a $\mathrm{Cu}$ X-ray lamp, and an Oxford Instruments Ltd. vibrating sample magnetometer (VSM).

\section{Results and discussion}

The electron microscopy measurements, whose results are shown in Fig. 1, have been applied in order to investigat the morphological and structural properties of the as-prepared iron-nickel nanomaterials.

Analysing the recorded images, one can see that both $\mathrm{Fe}-\mathrm{Ni}$ samples exhibit quite complex architectures. In general, they comprise of nanoparticles aligned in almost straight chains. Besides that, they reveal a typical core-shell structures where a dark core region and an ultra-thin light-grey shell layer are clearly visible in TEM images for both nanostructures. Undoubtedly, this is related to a surface oxidation process which usually
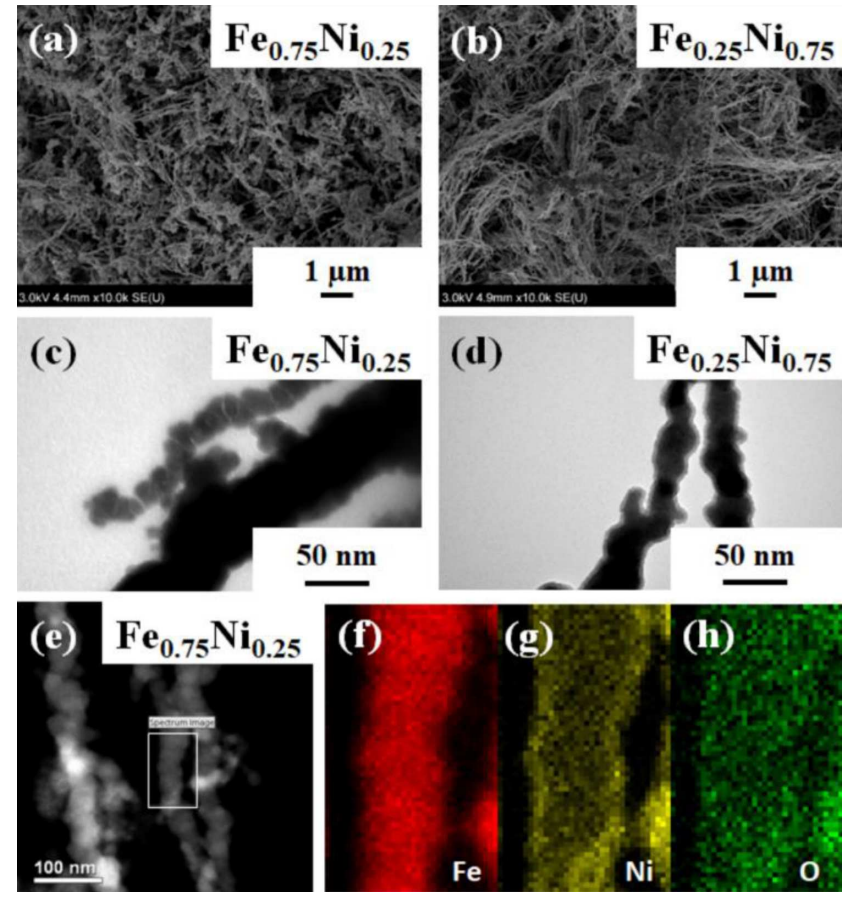

Fig. 1. (a, b) SEM images of $\mathrm{Fe}_{0.75} \mathrm{Ni}_{0.25}$ and $\mathrm{Fe}_{0.25} \mathrm{Ni}_{0.75}$ wire-like nanostructures. (c, d) TEM images of $\mathrm{Fe}_{0.75} \mathrm{Ni}_{0.25}$ and $\mathrm{Fe}_{0.25} \mathrm{Ni}_{0.75}$ wire-like nanostructures. (e) TEM image of $\mathrm{Fe}_{0.75} \mathrm{Ni}_{0.25}$ wire-like nanostructures with a marked rectangle area where the EDS elemental mapping of (f) $\mathrm{Fe},(\mathrm{g}) \mathrm{Ni}$, and (h) O atoms was performed. occurs in the nanomaterials containing $\mathrm{Fe}$ and/or Ni. At this point, it is worth noting that the thickness of oxide shell ranges between 2 and $4 \mathrm{~nm}$ and it is typical size for self-oxidized nanostructures [4-7]. According to the presented electron microscopy images, the sizes of diameters and lengths are inhomogeneously distributed for both $\mathrm{Fe}-\mathrm{Ni}$ samples. Their diameters vary between $20 \mathrm{~nm}$ and $130 \mathrm{~nm}$ but their average diameters equal $68 \mathrm{~nm}$ and $49 \mathrm{~nm}$ for $\mathrm{Fe}_{0.75} \mathrm{Ni}_{0.25}$, and $\mathrm{Fe}_{0.25} \mathrm{Ni}_{0.75}$ chains, respectively. In turn, the longer chains of about $2 \mu \mathrm{m}$ are found for the $\mathrm{Fe}_{0.25} \mathrm{Ni}_{0.75}$ sample, whereas the average length of $\mathrm{Fe}_{0.75} \mathrm{Ni}_{0.25}$ chains is around $1 \mu \mathrm{m}$.

The TEM observations have been additionally supplemented with the EDS elemental mapping measurements (cf. Fig. 1e) which indicate that the $\mathrm{Fe}-\mathrm{Ni}$ chains should be considered as alloy-type nanostructures because iron and nickel atoms forming investigated nanomaterials are well dispersed along their single chains. The elemental mapping also confirms that the samples are partially oxidized. Omitting the share of oxygen atoms, the estimated contributions of $\mathrm{Fe}$ and $\mathrm{Ni}$ atoms inside $\mathrm{Fe}_{0.75} \mathrm{Ni}_{0.25}$, and $\mathrm{Fe}_{0.25} \mathrm{Ni}_{0.75}$ chains agree well with the initial concentrations of particular ions taken to the reaction.

The structural investigations of the as-prepared Fe-Ni nanomaterials have been traced by means of powder X-ray diffraction (XRD), whose results are shown in Fig. 2. The presented patterns for both samples are almost similar. They consist of one low-intense narrow peak located at $69.7^{\circ}$, which can be assigned to $(400)$ plane of silicon holder [8], and two broad peaks. The pattern of $\mathrm{Fe}_{0.25} \mathrm{Ni}_{0.75}$ also reveals a peak at $30.5^{\circ}$ which according to JCPDS no. 73-2158 might be ascribed to one of the product of reaction, i.e., $\mathrm{H}_{3} \mathrm{BO}_{3}$. As stated above, the reflexes coming from the samples are very broad. This suggests that the investigated $\mathrm{Fe}-\mathrm{Ni}$ wire-like nanostructures are nanocrystalline or amorphous. In fact, this is in a good agreement with the previously synthesised Fe-Ni nanoparticles obtained by Douvalis et al. [5]. Furthermore, no signal related to the presence of iron oxides, nickel oxides, or iron-nickel oxides is registered. This indicates that the thin oxide shells observed in the TEM and EDS elemental mapping measurements are also amorphous.

The room temperature magnetization curves obtained for the randomly oriented $\mathrm{Fe}-\mathrm{Ni}$ wire-like nanostructures in the magnetic field up to $1 \mathrm{~T}$ are shown in Fig. 3. Their shapes are characteristic for the ferromagnetic materials. The saturation magnetizations $M_{S}$ measured for the $\mathrm{Fe}_{0.75} \mathrm{Ni}_{0.25}$ and $\mathrm{Fe}_{0.25} \mathrm{Ni}_{0.75}$ samples equal 86 and $10 \mathrm{~A} \mathrm{~m}^{2} / \mathrm{kg}$, respectively. These values agree more or less with the results obtained for the $\mathrm{Fe}-\mathrm{Ni}$ nanoparticles synthesised by Mokarian et al. [9]. The lower $M_{S}$ obtained for the $\mathrm{Fe}_{0.25} \mathrm{Ni}_{0.75}$ chains could be explained considering the higher content of $\mathrm{Ni}$ atoms in this sample which, indeed, reveal lower magnetic moment than Fe atoms. Besides that, according to the electron microscopy measurements, the diameters of $\mathrm{Fe}_{0.25} \mathrm{Ni}_{0.75}$ wire-like nanomaterial are lower than that for the $\mathrm{Fe}_{0.75} \mathrm{Ni}_{0.25}$ chains, 


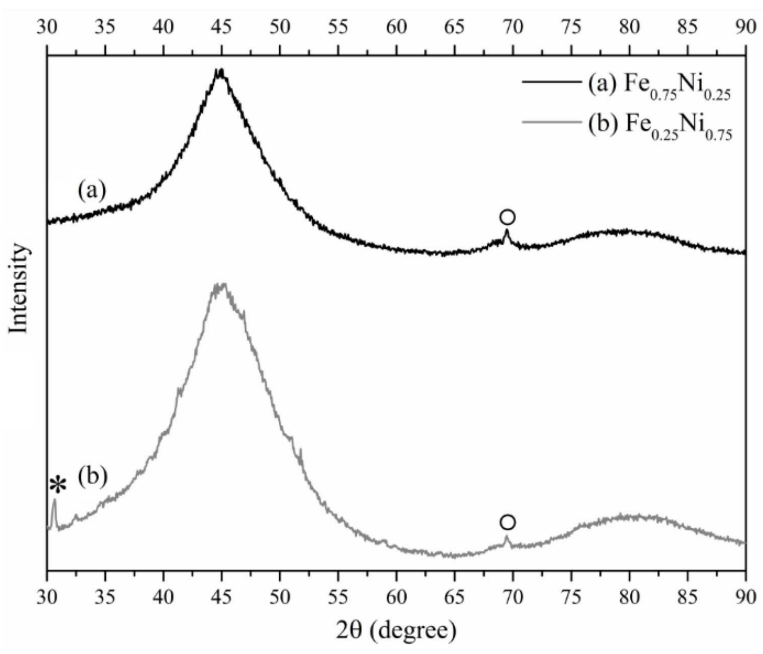

Fig. 2. XRD patterns of (a) $\mathrm{Fe}_{0.75} \mathrm{Ni}_{0.25}$ and (b) $\mathrm{Fe}_{0.25} \mathrm{Ni}_{0.75}$ wire-like nanostructures. The peaks position marked with asterisk and circles are assigned to contaminant $\mathrm{H}_{3} \mathrm{BO}_{3}$ (JCPDS no. 73-2158) and (400) plane of silicon holder (JCPDS no. 27-1402), respectively.

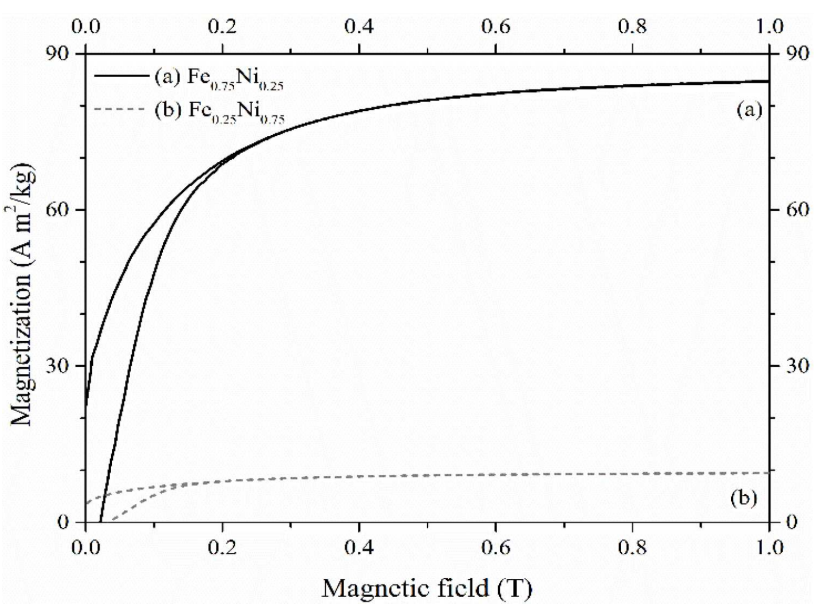

Fig. 3. Room temperature magnetization curves of (a) $\mathrm{Fe}_{0.75} \mathrm{Ni}_{0.25}$ and (b) $\mathrm{Fe}_{0.25} \mathrm{Ni}_{0.75}$ wire-like nanostructures.

whereas the thickness of oxide shell is almost the same for both nanomaterials. Therefore, it seems that the impact of oxide layer is more pronounced in the case of $\mathrm{Fe}_{0.25} \mathrm{Ni}_{0.75}$ chains. Moreover, the XRD patterns show that this sample is contaminated by $\mathrm{H}_{3} \mathrm{BO}_{3}$. Both these issues can result in the weakening of the magnetic response at the applied magnetic fields.

Analysing magnetization curves, it is also possible to observe that the coercivities $H_{C}$ for the $\mathrm{Fe}_{0.75} \mathrm{Ni}_{0.25}$ and $\mathrm{Fe}_{0.25} \mathrm{Ni}_{0.75}$ samples equal 17268 and $27852 \mathrm{~A} / \mathrm{m}$, respectively. This difference might be associated with dimensions of both samples and their core-to-shell ratios.
As stated above, the $\mathrm{Fe}_{0.25} \mathrm{Ni}_{0.75}$ chains are smaller than the $\mathrm{Fe}_{0.75} \mathrm{Ni}_{0.25}$ chains. Hence, assuming that the oxides present in the shell are harder magnetic materials than those in core, the $H_{C}$ increase can be referred to lower core-to-shell ratio for $\mathrm{Fe}_{0.25} \mathrm{Ni}_{0.75}$ sample.

\section{Conclusions}

The synthesis of the ferromagnetic bimetallic ironnickel wire-like nanostructures with $\mathrm{Fe}_{0.75} \mathrm{Ni}_{0.25}$ and $\mathrm{Fe}_{0.25} \mathrm{Ni}_{0.75}$ chemical compositions, in which the external magnetic field is applied as the growth parameter, is presented in this work. The as-prepared nanomaterials reveal quite complex structures. Namely, they are composed of $\mathrm{Fe}-\mathrm{Ni}$ nanoparticles aligned in almost straight chains. Moreover, they exhibit typical core-shell structures with the amorphous bimetallic alloy cores and very thin amorphous oxide shells. In this work, it is also shown that saturation magnetizations and coercivities of investigated nanostructures are dependent on their sizes and chemical compositions.

\section{Acknowledgments}

This work was supported by the National Science Centre (Poland) under the grant no. 2016/23/D/ST8/03268. Authors would like to thank Dr. Wen-An Chiou and Dr. Sz-Chian Liou from the Advanced Imaging and Microscopy Laboratory belonging to the University of Maryland for their support in the TEM and EDS measurements.

\section{References}

[1] M. Krajewski, Nanoscale 9, 16511 (2017).

[2] L. Tan, B. Liu, U. Glebe, A. Boker, Langmuir 34, 13993 (2018).

[3] Z.X. Kou, E. Liu, J.J. Yue, et al., J. Appl. Phys. 117, 17E709 (2015).

[4] M. Krajewski, W.S. Lin, H.M. Lin, K. Brzozka, S. Lewinska, N. Nedelko, A. Slawska-Waniewska, J. Borysiuk, D. Wasik, Beilstein J. Nanotechnol. 6, 1652 (2015).

[5] A.P. Douvalis, R. Zboril, A.B. Bourlinos, J. Tucek, S. Spyridi, T. Bakas, J. Nanopart. Res. 14, 1130 (2012).

[6] J.G. Railsback, A.C. Johnston-Peck, J.W. Wang, J.B. Tracy, ACS Nano 4, 1913 (2010).

[7] C.M. Wang, D.R. Baer, L.E. Thomas, J.E. Amonette, J. Antony, Y. Qiang, G. Duscher, J. Appl. Phys. 98, 094308 (2005).

[8] H. Meng, K. Fan, J.X. Low, J.G. Yu, Dalton Trans. 45, 13717 (2016).

[9] M.H. Mokarian, M. Almasi-kashi, S. AlikhanzadehArani, A. Ramazani, J. Mater. Sci. 52, 7831 (2017). 